This item was submitted to Loughborough's Research Repository by the author.

Items in Figshare are protected by copyright, with all rights reserved, unless otherwise indicated.

\title{
Development of an algorithm for automated cause-consequence diagram construction
}

PLEASE CITE THE PUBLISHED VERSION

PUBLISHER

(c) Inderscience Publishers

\section{VERSION}

AM (Accepted Manuscript)

\section{LICENCE}

CC BY-NC-ND 4.0

\section{REPOSITORY RECORD}

Valaityte, Akvilina, Sarah J. Dunnett, and J.D. Andrews. 2009. "Development of an Algorithm for Automated Cause-consequence Diagram Construction". figshare. https://hdl.handle.net/2134/5422. 
This item was submitted to Loughborough's Institutional Repository (https://dspace.lboro.ac.uk/) by the author and is made available under the following Creative Commons Licence conditions.

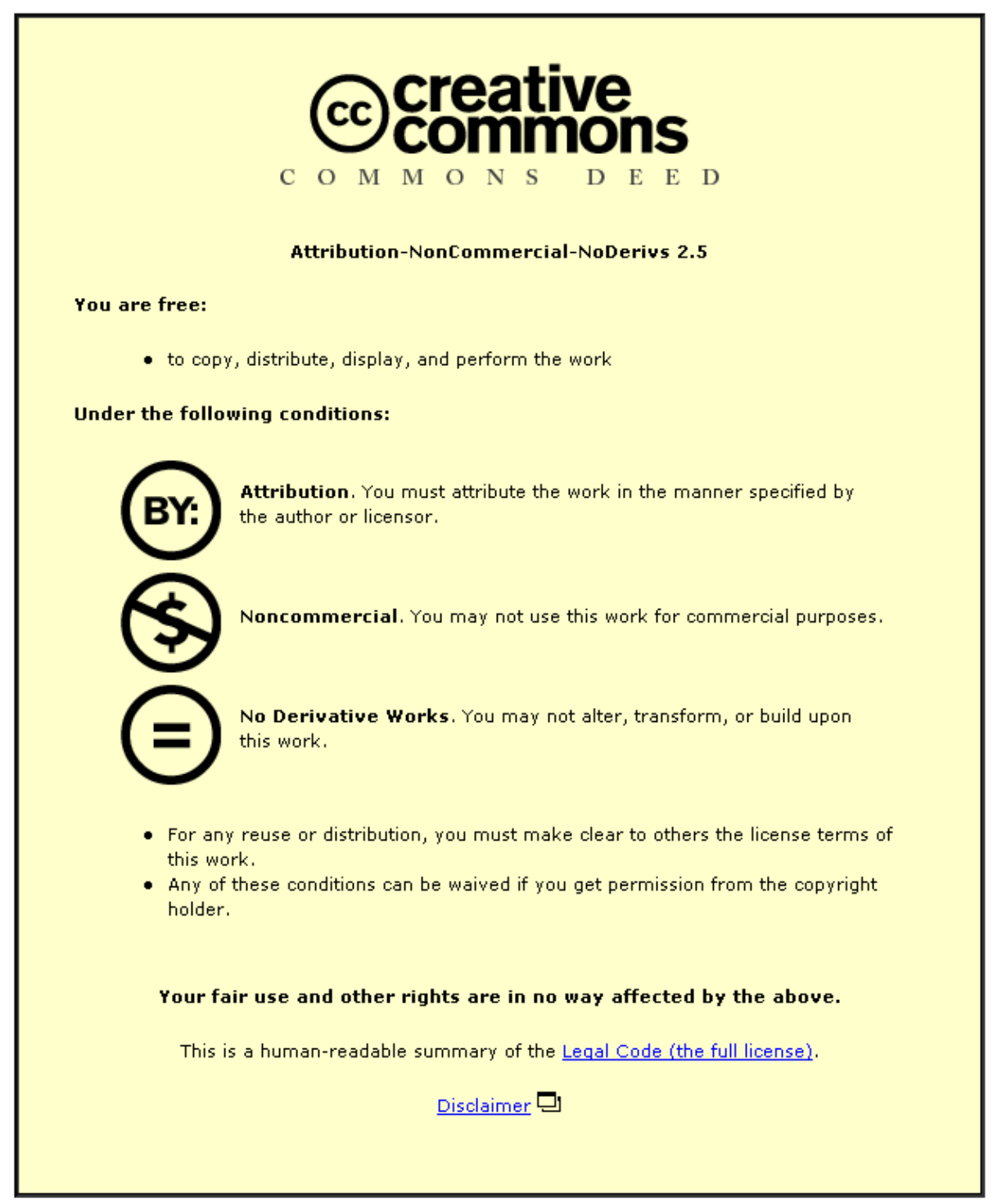

For the full text of this licence, please go to: http://creativecommons.org/licenses/by-nc-nd/2.5/ 


\section{Development of an algorithm for automated cause-consequence diagram construction}

\section{A Valaityte, S J Dunnett and J D Andrews}

Aeronautical and Automotive Engineering, Loughborough University, Loughborough, UK

Abstract: Cause-consequence analysis is one of the best tools available for a comprehensive reliability study. The cause-consequence diagram (CCD) method, like fault tree analysis, represents the failure logic of the system, but in addition the CCD also identifies the complete set of consequences following a given initiating event. Despite the clear appeal of this approach to engineers the CCD method has its drawbacks, e.g. for many industrial systems the diagram obtained will be very large and its final form dependant upon the analyst constructing it. If the diagram construction and its analysis could be automated it would reduce the potential for human error and increase the usefulness of the method.

Hence in this paper the development of an algorithm for automated cause-consequence diagram construction is described. The algorithm builds on methods developed previously for fault tree construction, such as topology diagrams describing how components are linked together in a system, component decision tables, used to model component behaviour, etc. Using this information a set of rules have been developed which enable automatic construction of the CCD. Once constructed the diagram can be quantified to give exact system reliability. To demonstrate the feasibility of the approach the algorithm is applied to a simple example.

Keywords: reliability, cause-consequence diagram, fault tree analysis 


\section{INTRODUCTION}

In his study, Nielsen [1] developed the cause-consequence diagram as a graphical tool for the analysis and description of relevant accidents in complex process plants. The method is based on a combination of standard reliability techniques. The cause diagram is the conventional fault tree used to describe all causes of an undesired event. The consequence diagram is an event-sequential diagram (decision-tree diagram) describing the alternative failure sequences that an abnormal event leads to if one or more of the accident preventing/limiting provisions fail. By using a combination of these reliability methods, the logical connections between independent accident causes and accident consequences can be established [2]. The main symbol in a CCD is a decision box which contains a component/subsystem condition. It is an identical representation of the YES/NO branches in an event tree. Following the YES/NO branches of the decision box the diagram is developed until it terminates in consequence boxes.

The CCD method has advantageous features in both its representation of a systems failure logic and its subsequent quantification. However traditional cause-consequence analysis is based on manual construction of the diagram. This requires a detailed knowledge of the system, plus experience and practice in understanding the failure modes of the components within the system and their effects. The construction is time-consuming and expensive, moreover, it can be a source of human errors. A faster and error free analysis can be performed if the CCD is automatically generated by computer from the system description [2].

Past work on automating reliability techniques has concentrated on fault tree analysis. As a result, a variety of methods for computerized fault tree synthesis have been developed and 
published. The most successful are based on the diagraph method [3] and the decision table method [4]. Lapp and Powers introduced a systematic algorithm to deal with control loops based on their digraph method [3]. The authors developed general operators whenever variables on the control loops were encountered in the digraph. A diagraph explicitly describes the relationships between process variables. The fault tree was then constructed by examining the cause and effect relationships indicated on the diagraph. Salem et al. [4] proposed a method to construct fault trees for general complex systems and implemented an automatic construction method called CAT (Computer Aided Tree). The components were modelled by decision tables describing the relations between the component inputs, component operation and failure modes, and the component outputs. A system topology was used to describe the connections between components. The features of diagraph and decision table methods were combined [5] in a new method for automating fault tree construction appropriate to modelling two-state and continuously variable safety systems. Since decision tables were used to model components behaviour no restriction was placed on the number of discrete states used to represent components behaviour. The gains, showing how changes in input parameters affect the output parameters, were incorporated into these tables enabling the method to deal with control loops.

In this paper previous methods applied to fault tree construction are extended to automate the construction of the CCD.

\section{GENERAL ALGORITHM}

In the following sections an algorithm will be described that accepts a description of the system under construction and generates a CCD which can be quantified in a straightforward manner. The automatic generation is based on the following steps: 
1. Initially information about the system required by the algorithm is given;

2. The CCD is then automatically constructed by applying the developed algorithm;

3. The CCD is reduced to its most efficient form by excluding irrelevant decision boxes;

4. Fault trees describing the failure of subsystems are constructed by employing the decision table method $[4,5]$;

5. The cause-consequence diagram is then used to analyse the system considered.

These five steps will be described in more detail in the following sections, 2.1 to 2.5.

\subsection{Algorithm requirements}

In order for the construction algorithm to be implemented detailed information about the system being considered must be given. This information is broken down into: component models, system topology diagram, and failure rate data for the components.

The component models used in this work are in the form of decision tables [4]. For each component its state is combined with all possible inputs from the components with which it is linked, to give outputs which are passed on to other components within the system. The construction of such tables and their use in other automated procedures is described in detail by Salem et al. [4].

The system topology diagram details how the components inputs and outputs are linked together within the system. 
The failure rate data includes a description of the failure modes of the components as well as their rate of failure. It is used in the construction of fault trees and their quantification, and hence the final CCD quantification.

For some systems it is also necessary to specify initial states for the components.

The algorithm also requires that an initiating event is identified. At the current stage of the algorithm development this event is given by the user. It depends upon the system under construction and is generally a component, with an associated function, which initiates the system. This is the first event considered in the algorithm.

In order to ensure that the CCD construction process completes and the algorithm does not continually consider components that are linked together, stopping criteria must also be identified. These indicate at which point the algorithm has reached a consequence and hence that path is completed.

A computer program implementing the construction algorithm is currently being developed. The end user will input the components within the system and how they are linked. The program will then draw upon a library of decision tables and construct the CCD diagram.

\subsection{Construction algorithm}

A set of steps have been developed to construct the consequence diagram. For ease of programming, systems have been classified into those that have circuits and those that don't. Where a circuit is defined as a path containing a power supply which starts and ends 
at the same component and with all components passing current. In the following subsections the steps for the two different cases have been summarised.

\subsubsection{Systems with circuits}

1. Initially, from the topology diagram, all possible paths through the system are traced. A path is obtained by tracing the output from a component to the input of the next until a component is repeated. From the list of possible paths the circuits are identified.

2. The algorithm proceeds by considering the component associated with the initiating event. The circuits are searched to determine which contain this component and then are considered in turn.

3. The decision table for the component being considered is investigated and for the given inputs the possible rows in the table identified. These are considered in turn. If the decision table includes functions these are checked to see if they can be applied. A decision box is created relating to the output in the row of the decision table being considered. The question in the decision box will relate to current/no current in the circuit under consideration.

4. The YES branch of the decision box is followed which determines the output in each component in the considered circuit. A check is made to see if any of the stopping criteria have been reached. If so the algorithm jumps to step 7 . If not a check is made to see if the circuit contains any components with external outputs which have not previously been considered and if so these are considered next. A 
components output is external if it is linked to a component which is not included in the circuit being considered. The decision table for that component is investigated and the relevant rows, taking into account previous decision boxes, established. A decision box is then added related to the external output. The YES branch of this box is then followed. If the circuit contains no components with external output that haven't already been considered the algorithm moves straight on to step 5 .

5. Once all components with external output have been considered the next circuit identified previously is considered in the same way by applying steps 3 and 4, until all circuits have been considered. If the decision table for the initial component contains functions which have not yet been applied then all circuits containing the component must be considered before these functions can be applied.

6. Once all circuits have been considered the decision table for the component under consideration is checked to see if any further functions can be applied. If so a decision box may be added related to the function. If not, the components with external outputs are reconsidered and the components to which their external output are linked identified. The circuits within which these components lie are then considered and steps 3, 4 and 5 applied. This continues until a stopping criteria is satisfied.

7. Once a stopping criteria is satisfied a consequence box is created showing the state of the system. A check is then made to determine if all decision boxes in the 
diagram have been developed completely. If so the diagram is complete, if not the algorithm moves onto the next step.

8. Backtrack to the last decision box and develop the NO branch in the same manner. This is continued until all decision boxes have been developed on both the YES and NO branches until a consequence box is reached.

\subsubsection{Systems without circuits}

1. Initially, using the topology diagram and the component decision tables, the order in which components and their output are considered is determined. Briefly this is achieved by starting with the component associated with the initiating event and considering all its outputs in turn. The components to which each of these outputs are connected are then considered and their outputs taken in turn, etc.

2. The algorithm proceeds by considering the first output of the component associated with the initiating event identified in step 1.

3. The components decision table is used to determine the possible values for the output and a decision box is added to the figure related to that value.

4. The YES branch is developed. A check is made to see if the stopping criteria have been satisfied. If so the algorithm jumps to step 7 . 
5. A check is made to see if any functions for the component under consideration can be applied. If so a decision box may be added if necessary related to the function and the algorithm returns to step 4 .

6. The next component output identified in step 1 is now considered and the algorithm returns to step 3 .

7. Once a stopping criteria is satisfied a consequence box is created showing the state of the system. A check is then made to determine if all decision boxes in the diagram have been developed completely. If so the diagram is complete, if not the algorithm moves onto the next step.

8. Backtrack to the last decision box and develop the NO branch in the same manner. This is continued until all decision boxes have been developed on both the YES and NO branches until a consequence box is reached.

The main steps for the diagram construction are represented in a flowchart for the 2 cases in Figures 1a and b, where the numbers in bold relate to the steps described above. 


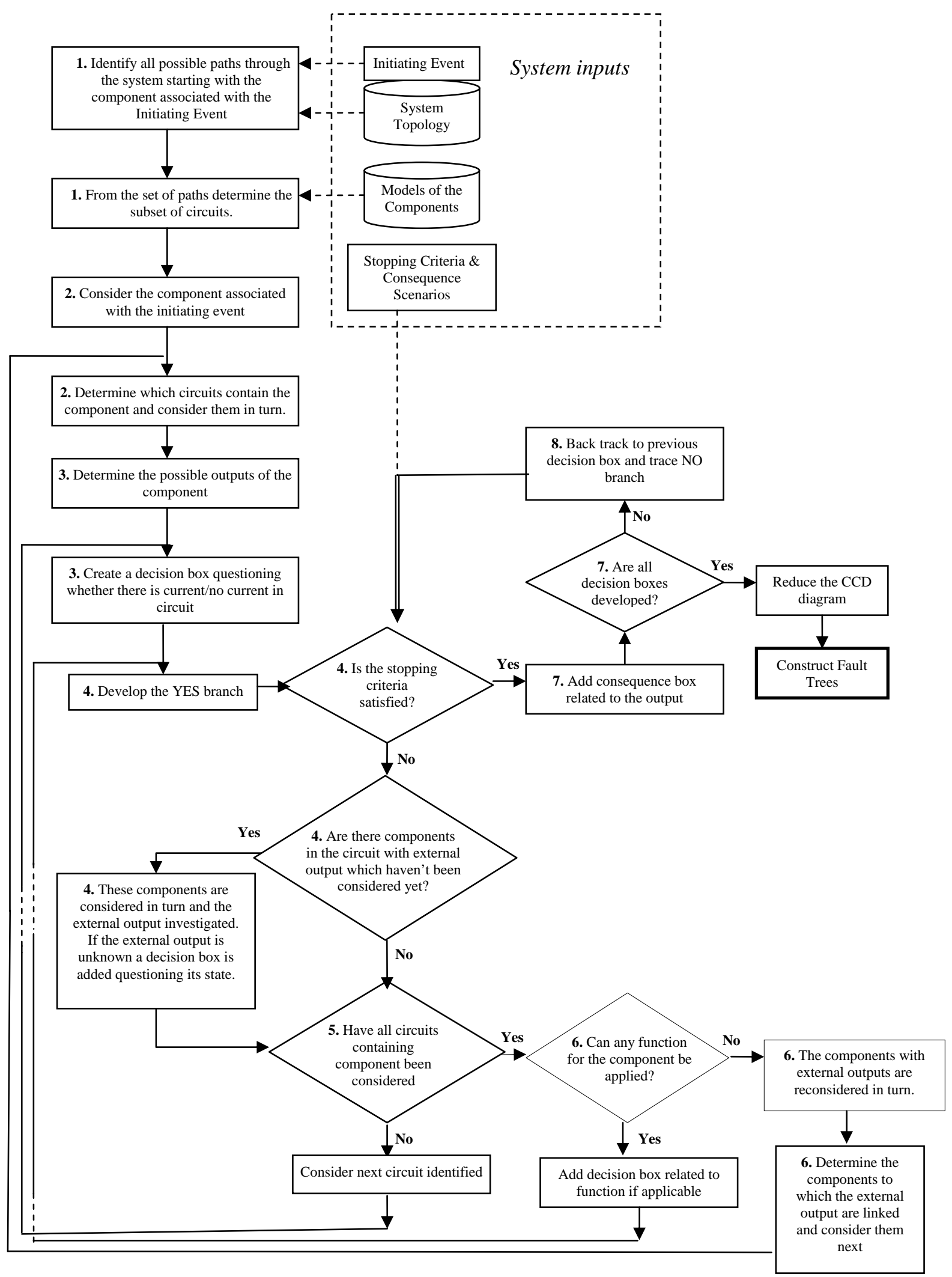

Fig. 1a Flowchart showing the main steps in the diagram construction for systems 


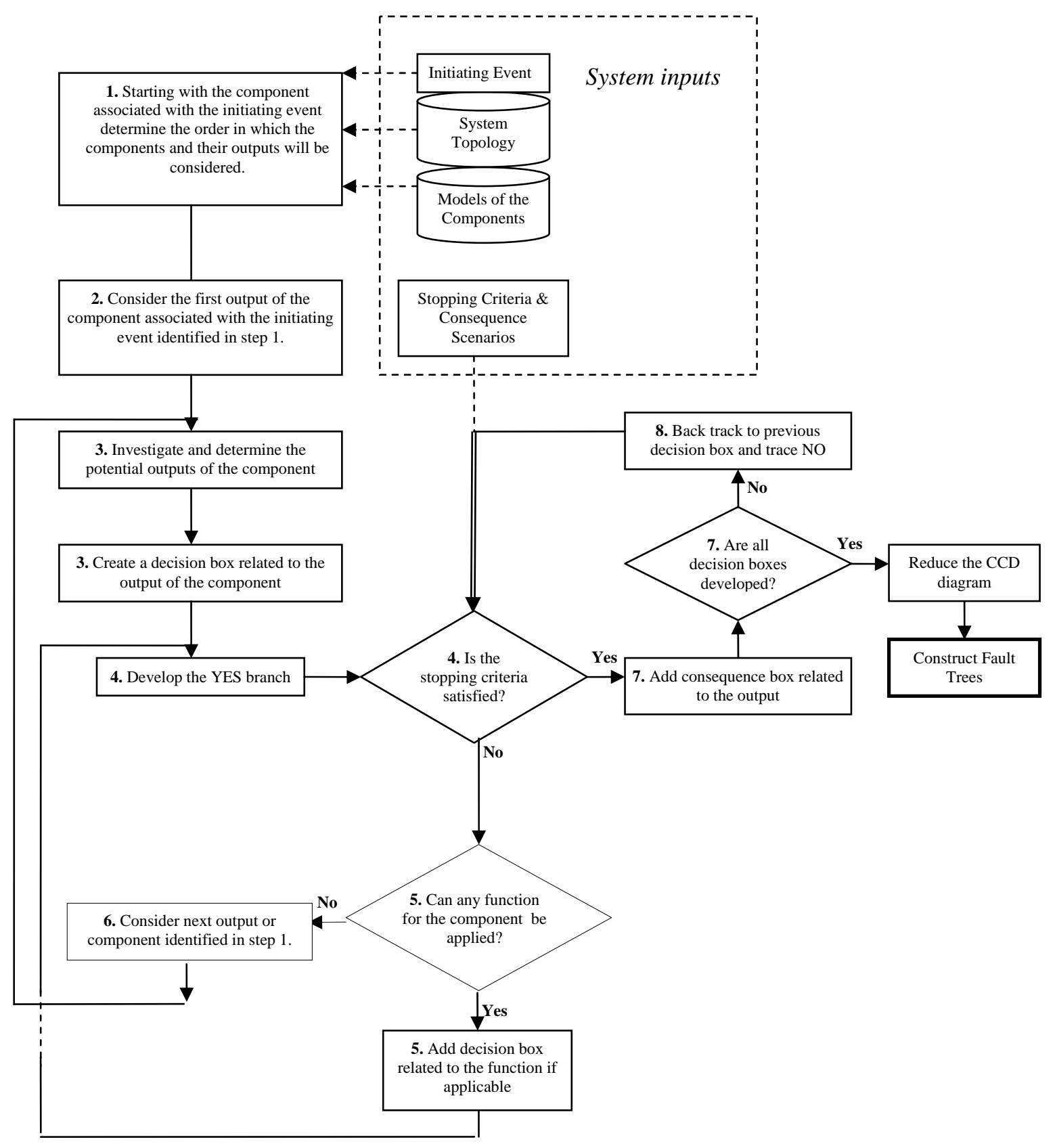

Fig. 1b Flowchart showing the main steps in the diagram construction for systems without circuits

In applying the steps outlined in sections 2.2.1 and 2.2.2 certain rules have to be applied.

The most commonly used ones are given below: 
1 First of all trace the YES branches of the decision boxes until a consequence box is reached. Then back track to the last decision box and develop the NO branch in the same way. Continue until the diagram is completed.

2 The output of the component being considered is obtained by taking the rows in its decision table in turn with the “don’t matter” cells considered last.

If the given inputs in a row of the decision table for the component considered, result in outputs which match previous ones considered, then the next row of the table is investigated. Otherwise consider the next function for this component.

3 If the component being considered has functions added to its decision table and it exists in more than one circuit and conditions exist such that a function is satisfied and hence applied. Then all the circuits in which the component exists must be considered before any further functions can be applied.

\subsection{Diagram reduction}

In this stage of the diagram generation it is reduced to its most efficient form. This is achieved by inspecting each decision box in the CCD. A decision box is deemed irrelevant if the boxes attached to its NO and YES branches are identical and their consequences are the same. Since the removal of the irrelevant box has no effect on the end result, it is excluded and the next decision box or consequence box in the path put in its place [6]. Figure 2 shows an example of an irrelevant decision box, box 2. In this example the state of component B is irrelevant as whichever path is taken from box 2 the outcome is the same. Since the state of component B is irrelevant, decision box 2 is removed, and then decision box 3 is encountered in its place and is renumbered. 


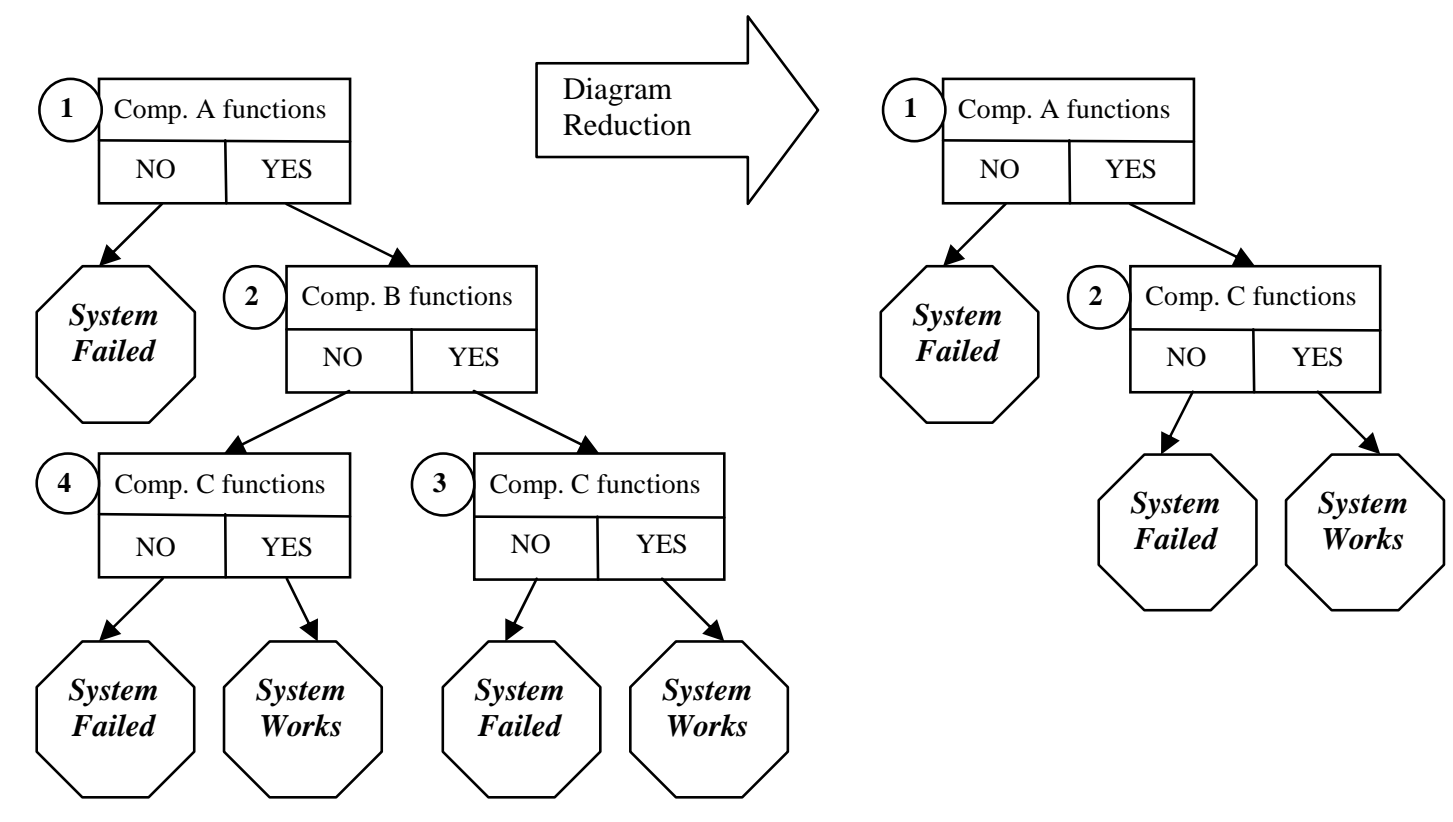

Fig. 2 Cause-consequence diagram reduction

If no further redundancies exist the cause-consequence diagram is deemed minimal [6].

\subsection{Development of the fault trees}

If a decision box is governed not by a component but by a sub-system then the probability of failure will be obtained via a fault tree [7]. These fault trees are produced automatically using fault tree construction methods developed previously [4, 5].

\subsection{Analysis}

Having constructed the CCD it can be used to analyse the system under consideration.

A qualitative analysis will produce the list of causes for each outcome condition. These are established by considering each decision box on the path to a particular outcome and 
listing the components failure or success as indicated by the exit path from the decision box.

A quantitative analysis will produce the probability of each system outcome. As the algorithm ensures that the probabilities of the decision boxes of the CCD are independent these are obtained by simply multiplying the probabilities of the component events in the branch leading to that consequence. The probability for each consequence for a system is then determined by summing the probability of each path leading to that consequence, as stated by Nielsen [1].

In order to demonstrate the construction and analysis process described above it is applied to a simple system.

\section{AUTOMATED CCD CONSTRUCTION EXAMPLE}

The cause-consequence diagram construction algorithm is now described in detail in relation to the pressure tank system illustrated in Figure 3. Initially the system is considered to be in a dormant state and thus de-energised. The switch (S), the relay contacts (C1 and C4) are all open, the timer (TIM) and pressure switch (PRS) contacts (C2 and C3) are closed. Depressing S provides power to the coil of relay 1 (R1) which results in the closure of contacts, C1. R1 self latches when S opens when released, and power is also supplied to relay 2 (R2) resulting in contacts, C4, closing which starts the motor (M) and hence pump (P). It is assumed that the tank (T) takes 30 minutes to fill and once the pressure threshold is reached the pressure switch opens contacts, C3, de-energising R2 which results in the removal of power from the pump motor. After a period of time the tank becomes empty and the pressure switch closes C3 which energises R2. The pump 
restarts and the filling process commences again. A safety feature - the timer - is included in case the pressure switch fails to open. When the contacts, C1, are closed power is supplied to the timer relay, this initiates a clock. If the clock registers 30 minutes of continuous pumping to the tank then the timer contacts, $\mathrm{C} 2$, are opened which results in a break in the circuit to R1 and system shutdown.

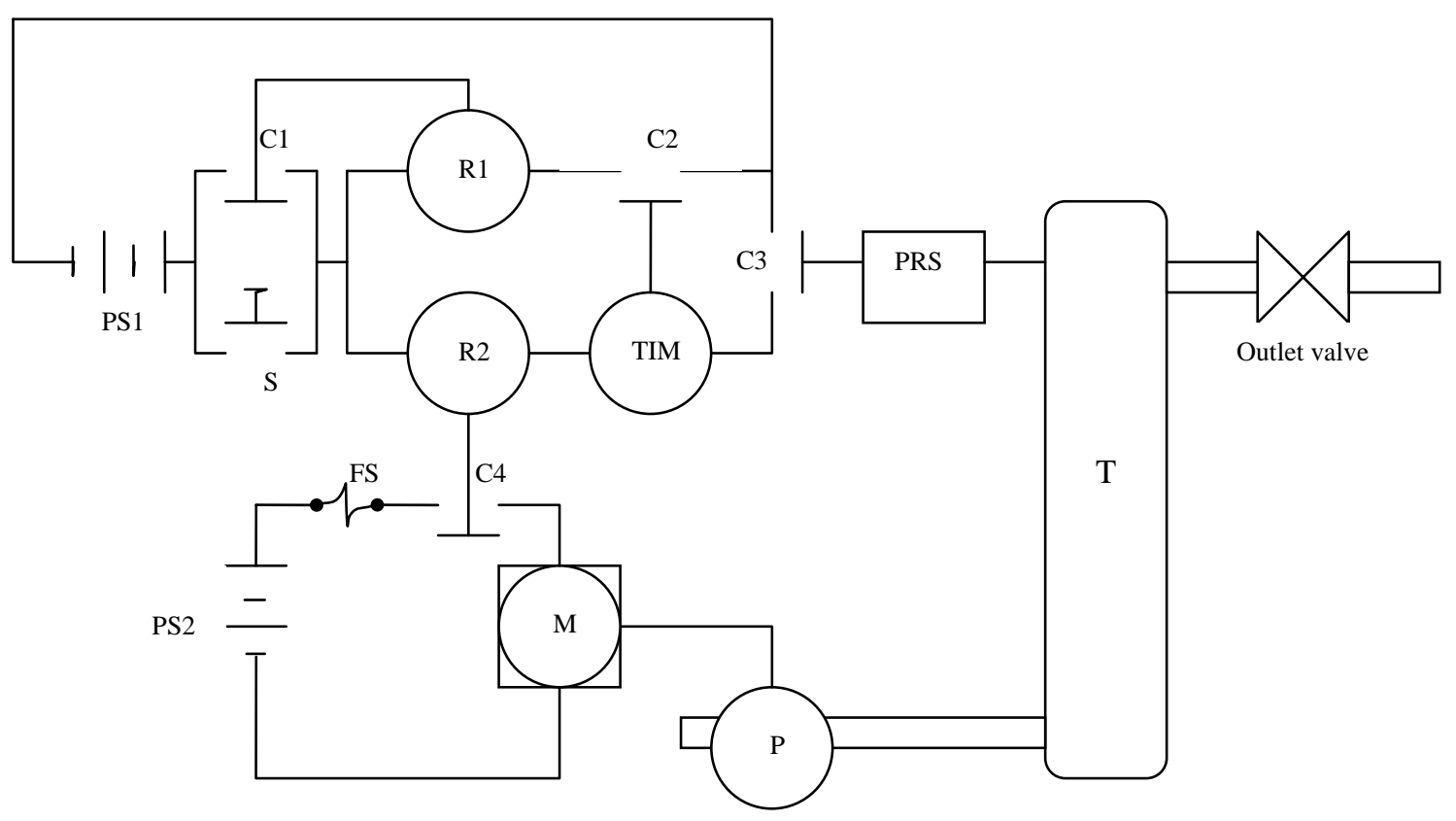

Fig. 3 Pressure tank system

\subsection{Automated CCD construction}

Following the automated algorithm described the following steps are taken:

i) Algorithm requirements: A topology diagram, showing how the components are linked together, is constructed, see Figure 4. In the diagram components are represented by 
rectangles. In order to construct this diagram it was necessary to use junctions J1-J4. These are parts of the system where information is sent in, or reduced from, more than one direction. For example, at J1 information from both $\mathrm{C} 1$ and $\mathrm{S}$ is returned to PS1. These junctions are labelled next to their position. For electrical systems, it is considered, that the current flows from right to left this is indicated by the arrows in the figure. 


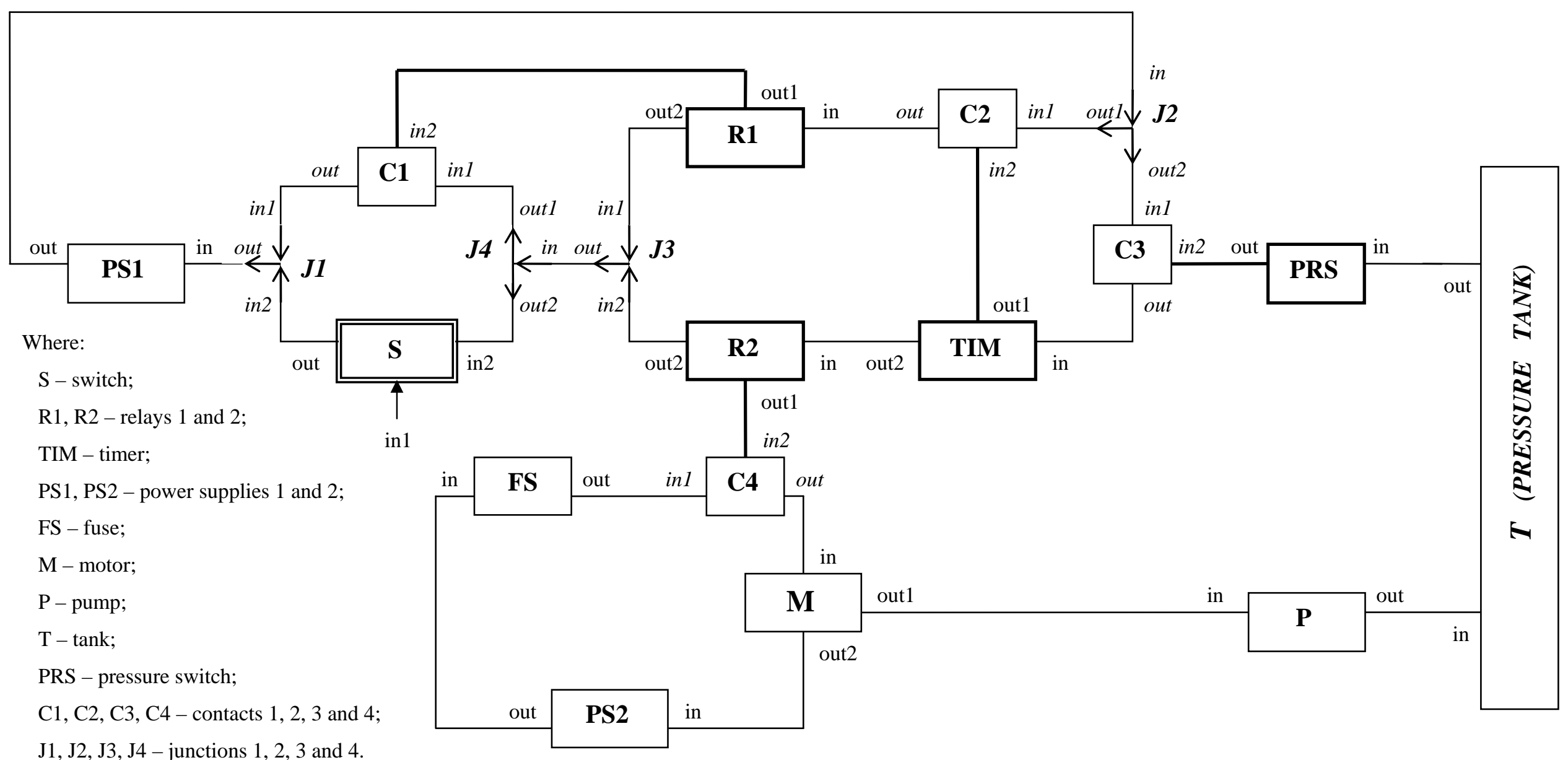

Fig. 4 Topology diagram for the pressure tank system 
The data for the components in the system is shown in Table 1. The failure data shown in the table are not real data but have been assumed here in order to demonstrate the technique described.

Table 1 Failure modes and failure data for the components

\begin{tabular}{|c|c|c|c|c|c|}
\hline Component & $\begin{array}{l}\text { Failure } \\
\text { mode }\end{array}$ & Description & $\begin{array}{l}\text { Failure } \\
\text { rate, } \lambda\end{array}$ & $\begin{array}{l}\text { Inspection } \\
\text { interval, } \theta\end{array}$ & $\begin{array}{l}\text { Mean time } \\
\text { to repair, } \tau\end{array}$ \\
\hline \multirow[t]{2}{*}{ Switch (S) } & S_FC & Switch failed closed & $1 \mathrm{E}-5$ & 4368.0 & 36.0 \\
\hline & S_FO & Switch failed open & $8.698 \mathrm{E}-4$ & - & - \\
\hline \multirow[t]{2}{*}{ Relay 1 (R1) } & R1_FC & Relay 1 fails closed & 2.3E-7 & 4368.0 & 36.0 \\
\hline & R1_FO & Relay 1 fails open & 2.3E-7 & - & - \\
\hline \multirow[t]{2}{*}{ Relay 2 (R2) } & R2_FC & Relay 2 fails closed & 2.3E-7 & - & - \\
\hline & R2_FO & Relay 2 fails open & 2.3E-7 & - & - \\
\hline \multirow[t]{2}{*}{ Timer (TIM) } & TIM_FC & Timer fails closed & $1 \mathrm{E}-5$ & 4368.0 & 36.0 \\
\hline & TIM_FO & Timer fails open & $1 \mathrm{E}-5$ & - & - \\
\hline \multirow[t]{2}{*}{$\begin{array}{l}\text { Pressure switch } \\
\text { (PRS) }\end{array}$} & PRS_FC & $\begin{array}{l}\text { Pressure switch } \\
\text { fails closed }\end{array}$ & $1 \mathrm{E}-5$ & - & - \\
\hline & PRS_FO & $\begin{array}{l}\text { Pressure switch } \\
\text { fails open }\end{array}$ & $1 \mathrm{E}-3$ & - & - \\
\hline Fuse (FS) & FS_F & Fuse fails broken & $1 \mathrm{E}-3$ & - & - \\
\hline $\begin{array}{l}\text { Power supply } 1 \\
\text { (PS1) }\end{array}$ & PS1_F & No power & $1 \mathrm{E}-3$ & - & - \\
\hline $\begin{array}{l}\text { Power supply } 2 \\
\text { (PS2) }\end{array}$ & PS2_F & No power & $1 \mathrm{E}-3$ & - & - \\
\hline Motor (M) & M_F & Motor fails broken & $1 \mathrm{E}-4$ & - & - \\
\hline Pump (P) & P_F & Pump fails broken & $1 \mathrm{E}-4$ & - & - \\
\hline Tank (T) & T_F & Tank fails broken & $1 \mathrm{E}-7$ & - & - \\
\hline
\end{tabular}

All the failure modes of the components in Table 1 are revealed except switch, relay 1 and timer, failed closed.

There are eleven decision tables relevant to the components in the example. These are shown in Tables 2 to 12. As an example of how these decision tables are constructed 
consider the switch, see Table 2. Two failure modes have been considered for this component, failed open $(F O)$ and failed closed $(F C)$ as described in Table 1. From the topology diagram, Figure 4, it can be seen that S has two inputs, IN1, - the manual closing or automatic opening and IN2 the current from junction J4. IN1 has two possible states, closed $(C L)$ and open $(O P)$, and IN2 has current $(C)$ or no current (NC). It has one output connected to IN2 of J1. Table 2 considers all possible combinations of inputs from IN1 and IN2 and all possible states of S and the effects these will have on the output to the J1. The sign "-”” in the inputs and state columns of the decision tables indicates the "don't matter" condition, which means that the specified input and component state will result in the specified output regardless of the value of the variable.

For example, for $\mathrm{S}$, there are two causes of current in the output, Table 2, these are:

1. Input 1 is closed $(I N 1=C L)$, current in input $2(I N 2=C)$ and switch working $(W)$, row 1.

2. Current in input $2(\operatorname{IN2}=C)$ and the switch failed closed $(F C)$, row 5 .

In order to model the fact that the switch only closes momentarily in this system, functions have to be added to the decision table, see Table 2. The first function states that:

1. If the switch is closed, working and the system has just started then the component remains closed and the time changes to $0.1 \mathrm{~min}$. This models the switch remaining closed for 0.1 minute. The second function states that:

2. If the switch is closed, working and the time is $0.1 \mathrm{~min}$ then the switch opens (IN1 => OP). This models the switch opening again after the time 0.1 minute has elapsed. 
The functions are investigated in turn and their effects investigated. Functions are added to decision tables in order to describe any time dependent behaviour. Some functions introduce process deviations while others may change the input or output variables of a certain component.

The other tables are constructed in a similar manner.

Table 2 Decision table for switch

\begin{tabular}{lcccc}
\hline & IN1 & IN2 & State & OUT \\
\hline 1 & $\mathrm{CL}$ & $\mathrm{C}$ & $\mathrm{W}$ & $\mathrm{C}$ \\
2 & $\mathrm{OP}$ & - & $\mathrm{W}$ & $\mathrm{NC}$ \\
3 & - & $\mathrm{NC}$ & - & $\mathrm{NC}$ \\
4 & - & - & $\mathrm{FO}$ & $\mathrm{NC}$ \\
5 & - & $\mathrm{C}$ & $\mathrm{FC}$ & $\mathrm{C}$ \\
\hline (1) & S: If (IN1 = CL $\cap$ State $=\mathrm{W} \cap$ Time $=0)$, \\
then IN1 $=\mathrm{CL} \cap$ Time $=0.1$ min; \\
(2) S: If (IN1 = CL $\cap$ State $=\mathrm{W} \cap$ Time $=0.1$ min), \\
\\
then IN1 => OP.
\end{tabular}

Table 3 Decision table for relay

\begin{tabular}{ccccc}
\hline & IN & State & OUT1 & OUT2 \\
\hline 1 & C & W & CL & C \\
2 & NC & W & OP & NC \\
3 & - & FO & OP & - \\
4 & - & FC & CL & - \\
5 & C & - & - & C \\
6 & NC & - & - & NC \\
\hline
\end{tabular}

Table 4 Decision table for timer

\begin{tabular}{ccccc}
\hline & IN & State & OUT1 & OUT2 \\
\hline 1 & C & W & CL & C \\
2 & NC & W & CL & NC \\
3 & - & FC & CL & - \\
4 & - & FO & OP & - \\
5 & C & - & - & C \\
6 & NC & - & - & NC \\
\hline (1) TIM: If (IN = C $\cap$ State $=\mathrm{W} \cap$ Time $>30$ min), \\
then OUT1 => OP.
\end{tabular}


Table 5 Decision table for power supply

\begin{tabular}{cccc}
\hline & IN & State & OUT \\
\hline 1 & $\mathrm{C}$ & $\mathrm{W}$ & $\mathrm{C}$ \\
2 & - & $\mathrm{F}$ & NC \\
3 & $\mathrm{NC}$ & - & NC \\
\hline
\end{tabular}

Table 6 Decision table for fuse

\begin{tabular}{cccc}
\hline & IN & State & OUT \\
\hline 1 & C & W & C \\
2 & - & F & NC \\
3 & NC & - & NC \\
\hline
\end{tabular}

Table 7 Decision table for motor

\begin{tabular}{ccccc}
\hline & IN & State & OUT1 & OUT2 \\
\hline 1 & $\mathrm{C}$ & $\mathrm{W}$ & ON & $\mathrm{C}$ \\
2 & $\mathrm{C}$ & $\mathrm{F}$ & OFF & $\mathrm{C}$ \\
3 & $\mathrm{NC}$ & - & OFF & NC \\
\hline
\end{tabular}

Table 8 Decision table for pump

\begin{tabular}{cccc}
\hline & IN & State & OUT \\
\hline 1 & ON & W & MF \\
2 & - & F & NMF \\
3 & OFF & - & NMF \\
\hline
\end{tabular}

Table 9 Decision table for tank

\begin{tabular}{cccc}
\hline & IN & State & OUT \\
\hline 1 & MF & W & LPR \\
2 & - & F & LPR \\
3 & NMF & - & LPR \\
\hline
\end{tabular}

(1) $\mathrm{T}$ : If (IN $=\mathrm{MF} \cap$ State $=\mathrm{W} \cap(0 \mathrm{~min}<$ Time $<30 \mathrm{~min})$ ), then OUT $=\mathrm{LPR} \cap$ Time $=>(=30 \mathrm{~min})$;

(2) $\mathrm{T}$ : If (IN $=\mathrm{MF} \cap$ State $=\mathrm{W} \cap$ Time $=30 \mathrm{~min})$, then $\mathrm{OUT}=>\mathrm{HPR} \cap$ Time $=>(>30 \mathrm{~min})$;

(3) $\mathrm{T}$ : If (IN $=\mathrm{MF} \cap$ State $=\mathrm{W} \cap$ Time $>30 \mathrm{~min})$, then OUT $=>$ VHPR.

Table 10 Decision table for pressure switch

\begin{tabular}{cccc}
\hline & IN & State & OUT \\
\hline 1 & LPR & W & CL \\
2 & HPR & W & OP \\
3 & - & FC & CL \\
5 & - & FO & OP \\
\hline
\end{tabular}


Table 11 Decision table for contacts

\begin{tabular}{cccc}
\hline & IN1 & IN2 & OUT \\
\hline 1 & C & CL & C \\
2 & - & OP & NC \\
3 & NC & - & NC \\
\hline
\end{tabular}

Table 12 Decision table for junctions

\begin{tabular}{ccccc}
\hline & IN1 & IN2 & OUT1 & OUT2 \\
\hline 1 & C & - & C & \\
2 & - & C & C & \\
3 & NC & NC & NC & \\
4 & C & & C & C \\
5 & NC & & NC & NC \\
\hline
\end{tabular}

Where $M F$ is mass flow, NMF is no mass flow, $L P R$ is low pressure, HPR is high pressure and VHPR is very high pressure. ON and OFF denotes motor turned on and off respectively. The blank cells in the decision table for the junctions 1-4, Table 12, are irrelevant. In this paper high pressure means the threshold pressure reached when the tank is full.

The initial conditions are that the switch and the relay contacts are open, timer and the pressure switch contacts are closed, i.e.

$\mathrm{S}: I N 1=O P$

R1: OUT1 = OP;

R2: $O U T 1=O P$;

TIM: OUT1 = CL;

PRS: $O U T=C L ;$

$\mathrm{T}: O U T=L P R$.

In order to start the algorithm the initiating component and its function must be identified. The system considered is initiated when the switch closes. Hence the 
initiating component is $\mathrm{S}$ with input 1 close $(C L)$. In the notation adopted here this is written as: S: $I N 1=>C L$ and the time is set to zero $($ Time $=0)$.

The stopping criteria in this case is that all the components in the system which have the capability to change their outputs have been considered. There are three possible outcomes to consider, when the tank indicates:

- $\quad$ low pressure, i.e. the tank is empty, $\mathrm{T}$ : $(O U T=>L P R)$;

- $\quad$ high pressure, i.e. the system operates normally or safe, $\mathrm{T}$ : $(O U T=>H P R)$;

- very high pressure, the failure of some components leads to continuous pumping to the tank and hence its overpressure, i.e. $\mathrm{T}$ : $(O U T=>V H P R)$.

ii) Construction algorithm: The CCD is constructed in the manner described in section 2.2.1 and shown schematically in Figure 1a.

Step 1: Five circuits are identified in this example:

1) $\{\mathrm{PS} 1, \mathrm{~J} 2, \mathrm{C} 2, \mathrm{R} 1, \mathrm{~J} 3, \mathrm{~J} 4, \mathrm{C} 1, \mathrm{~J} 1, \mathrm{PS} 1\}$;

2) $\{\mathrm{PS} 1, \mathrm{~J} 2, \mathrm{C} 2, \mathrm{R} 1, \mathrm{~J} 3, \mathrm{~J} 4, \mathrm{~S}, \mathrm{~J} 1, \mathrm{PS} 1\}$;

3) $\{$ PS1, J2, C3, TIM, R2, J3, J4, C1, J1, PS1 ;

4) $\{\mathrm{PS} 1, \mathrm{~J} 2, \mathrm{C} 3, \mathrm{TIM}, \mathrm{R} 2, \mathrm{~J} 3, \mathrm{~J} 4, \mathrm{~S}, \mathrm{~J} 1, \mathrm{PS} 1\}$;

5) $\{$ PS2, F, C4, M, PS2 $\}$.

Step 2: Since the switch is the initiating component, circuits 2 and 4 are considered as they contain S. Circuit 2 will be considered first. 
Step 3: The initiating event is $I N 1=C L$. Considering the decision table for S, Table 2, the algorithm searches for the rows whose first column for the input IN1 could take the value $C L$. These are rows $1,3,4$ and 5 . Considering these in order, row 1 is considered first, hence $I N 2=C$, State $=W$, OUT $=C$. Considering the functions attached to Table 2 it can be seen that function 1 is now satisfied and hence Time $=$ $0.1 \mathrm{~min}$. After a function has been applied all the circuits must be considered before applying any further functions in turn, see rule 4 . Decision box 1 is created related to the output $O U T=C$, see Figure 5a: "Is C in Circuit 2?” i.e. does the switch close and the power supply work?

Step 4: The YES branch of the decision boxes is followed which results in current in the 2nd circuit. In this case considering the components within circuit 2 there is current in:

- $\quad$ OUT of J1 (since $C$ is in IN2 to J1 and IN1 is unknown, row 2 in Table 12 describes the situation);

- $\quad$ OUT of PS1 (row 1 in Table 5);

- $\quad$ OUT1 of J2 (row 4 in Table 12);

- $\quad$ OUT of C2 (row 1 in Table 11);

- $\quad$ OUT2 of R1 (rows 1, 3-5 in Table 3);

- $\quad$ OUT of J3 (row 1 in Table 12) and

- $\quad$ OUT2 of J4 (row 4 in Table 12).

The stopping criteria has not been satisfied so a check is made of the components in circuit 2 to determine whether any have external output. One such component is identified, R1, as the output from this component is linked to an input of C1 which is in circuits 1 and 3 but not 2 . 
The decision table for the relay, Table 3, is now considered. As it has been assumed that there is current in the circuit 2, (YES branch of decision box 1, Figure 5a), four rows of the table satisfy this (rows $1,3-5$ ). However, in the 4th row the relay is in a failed closed state, which contradicts the initial assumption that the relay is opened and hence this row is not relevant. The column related to the external output is investigated. Considering rows 1,3 and 5 in Table 3 in turn, row 1 is considered first. In this case OUT1 $=C L$. Hence the decision box 2 is added which contains the question “Is R1: OUT1 = CL?”, see Figure 5a.

Step 5: As there are no more components with external output in circuit 2 any other circuits containing $\mathrm{S}$ must be considered. Hence circuit 4 is investigated next. A decision box should be created asking whether there is current, or no current in the circuit. However from the path taken (YES branch of decision box 1) and the initial condition for PRS it is known that there is current in circuit 4 and hence this box is unnecessary. Hence any components within the 4th circuit with external output are considered. There are two such components: timer, TIM, and relay 2, R2. The external output OUT1 of the TIM connects to input IN2 of contacts 2, C2, which is in circuits 1 and 2 but not 4 . Since there is current in circuit 4 rows 1,3 and 5 of the decision table for the timer are considered (row 4 is not considered as initially the timer was closed and hence it cannot be in a FO state), see Table 4. Row 1 is considered first, this gives OUT1 $=C L$, which coincides with the initial state for this output. As this results in no change to the system rule 3 is applied. Hence the third row of Table 4 is considered next. However, this also gives OUT1 $=C L$ and therefore row 5 is considered. In this case the value of OUT1 doesn't matter and hence is not relevant. As there are no rows left in Table 4 with $I N=C$, the algorithm searches for 
any added functions for this component, by rule 3. There is one added function but the conditions for it are not satisfied and hence the next component that is considered is R2. A decision box 4 is created which relates to the entry of OUT1 in Table 3 (same as for R1), see Figure 5a.

Step 6: As the two circuits containing S, circuits 2 and 4, have now been considered the decision table for S, Table 2, is checked to see if there are any functions which can be applied. In this case function (2) needs to be considered. A decision box is created related to the switch, as $I N 1=C L$ and Time $=0.1 \mathrm{~min}$, from earlier. The only unknown condition is its state (either $W$ or $F C$, see Table 2). Thus the decision box 4 in Figure 5a asks “Is S: OUT => NC?”. Therefore, following the YES branch, from the decision table it can be seen that the switch works and consequently after 0.1 minutes opens.

Now all components with external output and functions in circuits 2 and 4 have been investigated. The construction algorithm proceeds by reconsidering the components with external output, R1, TIM and R2, and the components to which their external outputs are linked are investigated. Considering initially R1, as OUT1 is connected to IN2 of C1, these contacts, and therefore circuits 1 and 3, are considered next in turn.

Steps 3, 4 and 5 are then repeated for this component. The component to which the external output of TIM is connected is then considered etc., until a stopping criteria is reached, i.e. all the components which could change their outputs have been considered. This occurs, on the YES branches, after decision box 10, see Figure 5a. 
Step 7: A consequence box is created showing the state of the system which in this case is that the tank indicates high pressure, $\mathrm{T}: O U T=H P R$.

Step 8: The rest of the CCD is obtained in the same manner by back tracking to the last decision box and developing the NO branch until the cause-consequence diagram is complete.

iii) Diagram reduction: The cause-consequence diagram presented in Figures 5a, b is in its reduced form.

iv) Development of the fault trees: Fault trees, shown in Figure 6, are now created using previously developed methods, see [4] for each decision box.

v) Analysis: The final cause-consequence diagram for the pressure tank system, Figure 3, is presented in Figures 5a, b with the corresponding fault trees in Figure 6. The diagram is in a form where each path contains independent events in the decision boxes and can be quantified with ease. 


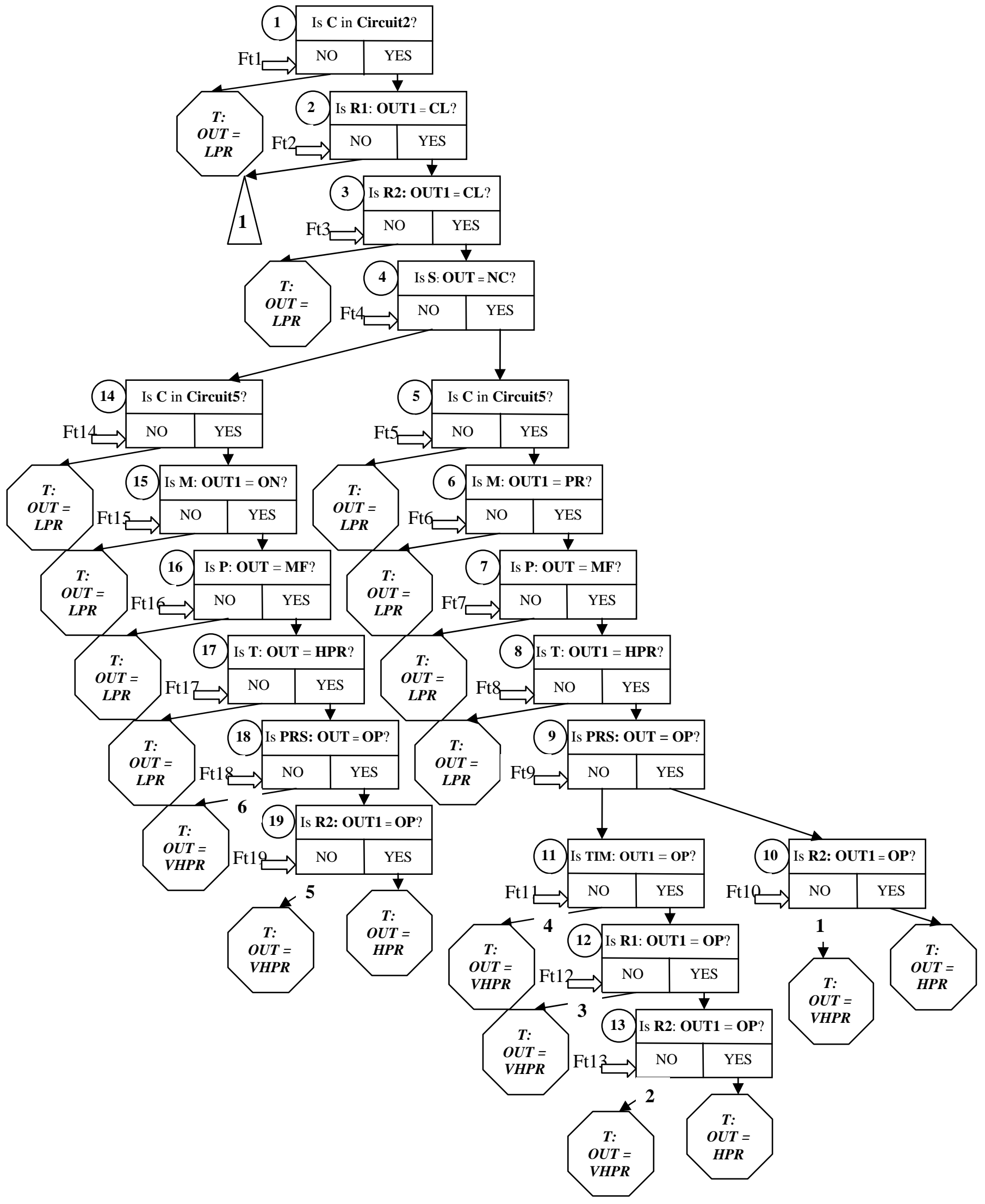

Fig. 5a Cause-consequence diagram for pressure tank system 


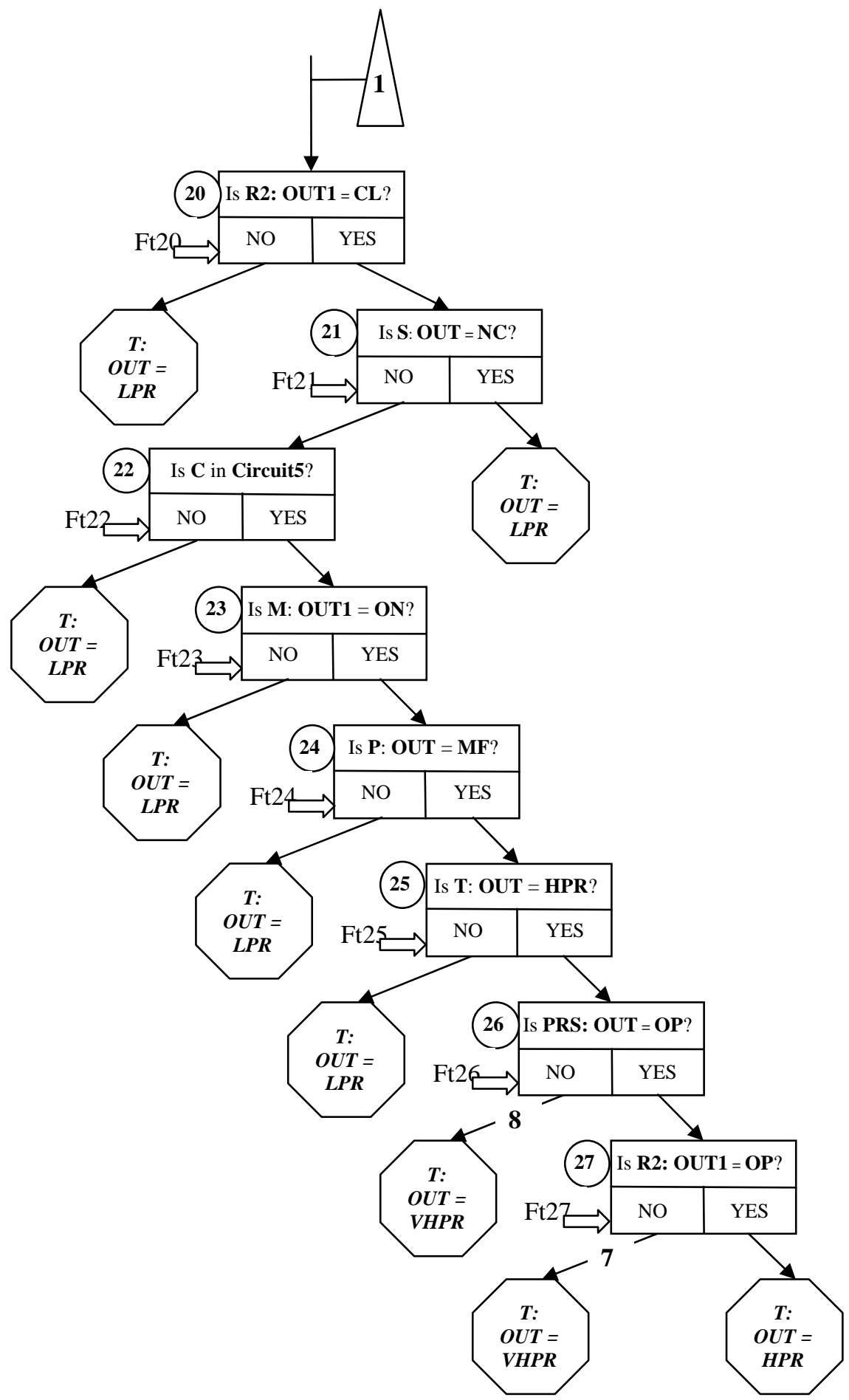

Fig. 5b Cause-consequence diagram for pressure tank system (continued) 

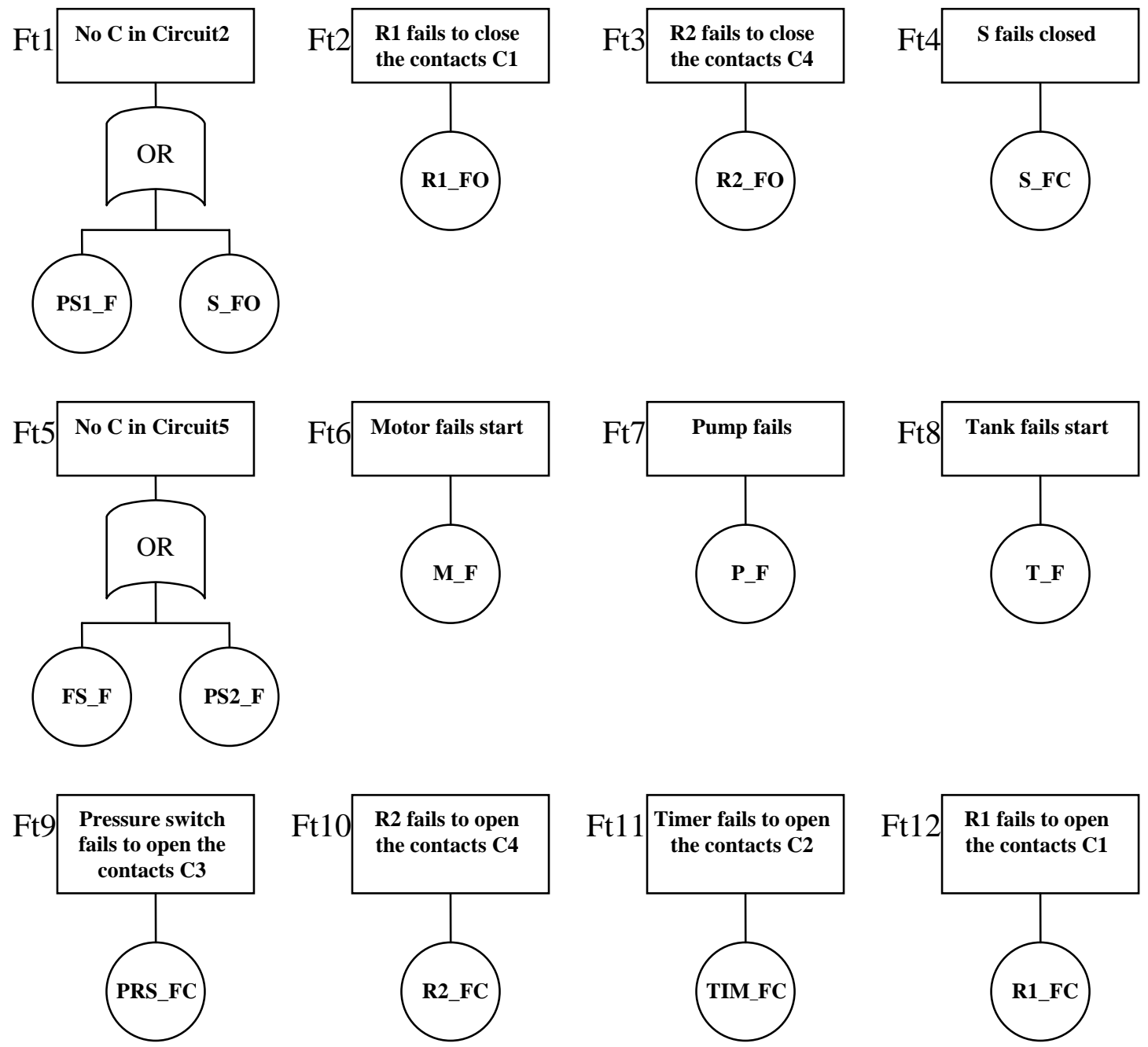

$$
\begin{aligned}
& \text { Ft13 }=\text { Ft10 }=\text { Ft19 }=\text { Ft } 27 ; \\
& \text { Ft14 }=\text { Ft5 }=\text { Ft22; } \\
& \text { Ft15 }=\text { Ft6 = Ft23; } \\
& \text { Ft16 }=\text { Ft7 = Ft24; } \\
& \text { Ft17 }=\text { Ft8 = Ft25; } \\
& \text { Ft18 }=\text { Ft9 = Ft26; } \\
& \text { Ft20 }=\text { Ft3 } \\
& \text { Ft21 }=\text { Ft4 }
\end{aligned}
$$

Fig. 6 Fault trees for the CCD shown in Figures 5a, b. 


\subsection{Qualitative analysis}

For the example considered the failure event is very high pressure in the tank, i.e. the consequence "T: OUT $=V H P R$ ". In the diagram there are eight consequence boxes with this outcome, these are numbered 1-8 on Figures 5a, b. To obtain the minimal cut sets the failure events leading to these boxes must be traced. For example, for the consequence box 1 on the NO branch of decision box 10 the component failures leading to this are given from Ft10, i.e. R2_FC. Considering the other consequence boxes in the same manner and disregarding any non-minimal contributions, leads to the complete list of minimal cut sets:

$$
\{\text { R2_FC }\},\{\text { PRS_FC, R1_FC }\},\left\{P R S \_F C, T I M \_F C\right\},\{\text { S_FC, PRS_FC }\} \text {. }
$$

\subsection{Quantitative Analysis}

Considering the paths of the CCD leading to VHPR the probability of the system failing and producing very high pressure can be obtained. There are 8 such mutually exclusive paths. The first one leading to the consequence "T: OUT =VHPR", numbered 1 on the diagram, is: YES branches of decision boxes 1-9 and NO branch of decision box 10 . The probability of the YES branch of decision box 1 is $\left(1-q_{F t 1}\right)$ etc. Hence the probability of path 1 is given by:

$$
\begin{aligned}
P(\text { Path } 1)= & \left(1-q_{F t 1}\right)\left(1-q_{F t 2}\right)\left(1-q_{F t 3}\right)\left(1-q_{F t 4}\right)\left(1-q_{F t 5}\right)\left(1-q_{F t 6}\right)\left(1-q_{F t 7}\right) . \\
& \cdot\left(1-q_{F t 8}\right)\left(1-q_{F t 9}\right) q_{F t 10}
\end{aligned}
$$

where $q_{F t 1}$ is the probability of the top event of fault tree 1 etc. Using the fault trees shown in Figure 6 this can be written in terms of the basic events as:

$$
\begin{aligned}
P(\text { Path } 1)= & \left(1-q_{S_{-} F O}-q_{P S 1_{-} F}+q_{S_{-} F O} q_{P S 1_{-} F}\right)\left(1-q_{R 1_{-} F O}\right)\left(1-q_{R_{-} F O}\right) . \\
& \cdot\left(1-q_{S_{-} F C}\right)\left(1-q_{F S_{-} F}-q_{P S 2_{-} F}+q_{F S_{-} F} q_{P S 2_{-} F}\right)\left(1-q_{M_{-} F}\right) . \\
& \cdot\left(1-q_{P_{-} F}\right)\left(1-q_{T_{-} F}\right)\left(1-q_{P R S_{-} F C}\right) q_{R_{2} 2_{-} F}
\end{aligned}
$$


where $q_{S_{-} F O}$ is the probability of the switch failing open etc. The probabilities of the other paths are obtained in the same manner. Assuming that the pressure tank becomes empty after 12 hours, the probability of failure for revealed failures is obtained using Equation 1 with $t=12$ hours :

$$
q=1-\mathrm{e}^{-\lambda t}
$$

For unrevealed failures the probability of failure is obtained using $\theta$ and $\tau$, given Equation 2:

$$
q=\lambda \cdot\left(\frac{\theta}{2}+\tau\right)
$$

The probability of $V H P R$ is obtained by summing the probabilities of all of these paths. Therefore:

$$
\mathrm{P}(\text { Very high pressure in the tank })=\sum_{i=1}^{8} \mathrm{P}(\text { Path } i) \text {. }
$$

Using the data given in Table 1 and Equations 1-3 the probability of very high pressure in the tank is found to be $8.0871 \cdot 10^{-6}$. This is the same result as would be obtained by taking the four minimal cut sets listed earlier and using the inclusionexclusion expansion [8].

\section{CONCLUSIONS}

An algorithm to automatically construct cause-consequence diagrams is developed in this paper. From an initial description of a system the algorithm develops the CCD in a logical manner. Such an automatic construction saves an analyst from a laborious and time consuming task and ensures that all possible system behaviour is modelled. The diagram generated can be quantified simply to give exact probabilities of all 
system outcomes considered. The algorithm employs topology diagrams and decision tables, methods developed previously for fault tree construction.

In order to demonstrate the algorithm a pressure tank example has been considered here, however the algorithm has been tested with other substantial industrial examples.

\section{REFERENCES}

1. Nielsen, D.S. Use of cause-consequence charts in practical systems analysis. Reliability and Fault Tree Analysis, 1975, 849-880.

2. Nielsen, D.S. and Runge, B.A. Unreliability of a standby system with repair and imperfect switching. IEEE Transactions on Reliability, 1974, 23(1), 17-24.

3. Lapp, S.S. and Powers, G.J. Computer-aided synthesis of fault trees. IEEE Transactions on Reliability, 1977, 26(1), 2-13.

4. Salem, S.L., Apostolakis, G.E. and Okrent, D. A computer-oriented approach to fault tree construction. EPRI Report, 1976, np-288.

5. Henry, J.J. and Andrews, J.D. A computerised fault tree construction methodology. Proc. IMechE, Part E, Journal of Process Mechanical Engineering, 1997, 211(3), 171-185.

6. Andrews, J.D. and Ridley, L.M. Reliability of sequential systems using the cause-consequence diagram method. Proc. IMechE, Part E, Journal of Process Mechanical Engineering, 2001, 215(3), 207-220.

7. Andrews, J.D. and Ridley, L.M. Application of the cause-consequence diagram method to static systems. Reliability Engineering and System Safety, 2002, 75(1), 47-58. 
8. Andrews, J.D. and Moss, T.R. Reliability and risk assessment (2nd ed.) 2002 (Professional Engineering Publishing, Bury St Edmunds and London).

\section{APPENDIX}

\section{Table Captions}

Table 1 Failure modes and failure data for the components

Table 2 Decision table for switch

Table 3 Decision table for relay

Table 4 Decision table for timer

Table 5 Decision table for power supply

Table 6 Decision table for fuse

Table 7 Decision table for motor

Table 8 Decision table for pump

Table 9 Decision table for tank

Table 10 Decision table for pressure switch

Table 11 Decision table for contacts

Table 12 Decision table for junctions

\section{Figure captions}

Fig. 1a Flowchart showing the main steps in the diagram construction for systems containing circuits

Fig. 1b Flowchart showing the main steps in the diagram construction for systems without circuits

Fig. 2 Cause-consequence diagram reduction 
Fig. 3 Pressure tank system

Fig. 4 Topology diagram for the pressure tank system

Fig. 5a Cause-consequence diagram for pressure tank system

Fig. 5b Cause-consequence diagram for pressure tank system (continued)

Fig. 6 Fault trees for the CCD shown in Figures 5a, b

$\begin{array}{ll}\text { Notation } & \\ q & \text { cause-consequence diagram } \\ \lambda & \text { failure probability } \\ \theta & \text { failure rate } \\ \tau & \text { inspection interval } \\ & \text { mean time to repair }\end{array}$

